\title{
Iliofemoral lysis for DVT remains ATTRACTive
}

\author{
Catherine Go, Efthymios D. Avgerinos \\ Division of Vascular Surgery, University of Pittsburgh Medical Center, Pittsburgh, PA, USA \\ Correspondence to: Efthymios D. Avgerinos, MD. Division of Vascular Surgery, University of Pittsburgh Medical Center, 200 Lothrop Street, Heart \\ and Vascular Institute, Room E351.1, South Tower, Pittsburgh, PA 15213, USA. Email: avgerinose@upmc.edu. \\ Provenance and Peer Review: This article was commissioned and reviewed by Academic Editor Zhiyuan Wu (Department of Vascular and Endovascular \\ Surgery, Klinikum rechts der Isar, Technical University Munich, Munich, Germany). \\ Comment on: Comerota AJ, Kearon C, Gu CS, et al. Endovascular thrombus removal for acute iliofemoral deep vein thrombosis. Circulation \\ 2019;139:1162-73.
}

Submitted Jul 21, 2019. Accepted for publication Aug 21, 2019.

doi: $10.21037 /$ cdt.2019.08.08

View this article at: http://dx.doi.org/10.21037/cdt.2019.08.08

Following the publication of a landmark paper on comparative effectiveness of anticoagulation against thrombolysis, the authors of the Acute Venous Thrombosis: Thrombus Removal with Adjunctive Catheter-Directed Thrombolysis (ATTRACT) trial released a subanalysis targeting the iliofemoral vein thrombosis, which excluded the femoropopliteal deep vein thrombosis (DVT) population. Similar to the primary publication, the ATTRACT-iliofemoral results again challenge the utility of pharmacomechanical thrombolysis (PMT) and catheterdirected thrombolysis (CDT) in preventing post-thrombotic syndrome (PTS) $(1,2)$. Their analysis reported no difference in the development of PTS (defined as Villalta score $\geq 5$ ) between patients with proximal DVT treated with PMT/ $\mathrm{CDT}$ and anticoagulation alone [relative risk (RR), 0.95; 95\% confidence interval $(\mathrm{CI}), 0.78-1.15 ; \mathrm{P}=0.59$ ] at 24 months after treatment. This result contradicts that of their Norwegian contemporaries of the 2012 Catheter-directed Venous Thrombolysis (CaVenT) trial—a smaller randomized control trial which reported a lower rate of PTS in patients treated with CDT over anticoagulation alone (3).

Thrombolysis enthusiasts, however, need not retire their catheters just yet. Before delving into discussion about the primary outcome of the ATTRACT trial-the diagnosis of PTS-believers in PMT/CDT can rest assured that their interventions provide patients with a treatment that improves symptoms and quality of life (QOL). Short-term benefits of intervention include decreased leg pain and circumference at 10 and 30 days, while long-term outcomes include improved vein-related QOL at 24 months measured using VEINES-QOL scale (28.6 vs. 23.0, $\mathrm{P}=0.029)$. Most importantly though, was that PMT/CDT was associated with lower Villalta scores at every timepoint up to 24 months (3.70-3.95 vs. 5.38-5.54, $\mathrm{P}<0.01)$. Although there was no difference in patients with any diagnosis of PTS (Villalta score $\geq 5$ ), there were significantly fewer patients with moderate PTS (score $\geq 10,18 \%$ vs. $28 \%, \mathrm{P}<0.05$ ) or severe PTS (score $\geq 15,8.7 \%$ vs. $15.0 \%, \mathrm{P}<0.05$ ) when comparing 24-month outcomes of PMT/CDT vs. control (2). The advantages of intervention are clear, and although the ATTRACT trial suggests there is no preventative benefit against PTS, one must take pause and consider the limitations of the study.

The ATTRACT trial initially aimed to include only patients with iliofemoral DVT. However, due to slow enrollment, the authors added patients with femoropopliteal DVTs. Clearly, the ATTRACT team was not satisfied with the number of iliofemoral patients that were enrolled and added the femoropopliteal cohort to reach their statistical power goal. Performing an analysis of the iliofemoral subset of this sample inherently decreases the power to detect differences between groups. Other sampling factors may also be playing a role. In the original ATTRACT trial, the screening to enrollment ratio was 41 to 1 (4). Over 26,000 patients met inclusion criteria but were not randomized, and 1,100 patients declined randomization. Who were these patients? Presumably, some of these patients had symptoms so severe that physicians or patients themselves would not consider randomization-feeling confident that intervention would likely be more advantageous (5). 
Another aspect of the study that may inspire caution is the heterogeneity of the intervention arm and how it deviates from contemporary techniques. Catheter-directed lytic infusion is a well-established procedure performed routinely with minimal variation in technique, but almost half of the intervention arm instead received PMT using two devices that were relatively new to the market at the time of the trial. One should note that one of these devices is no longer even available for use! The ATTRACT trial reports an $86 \%$ thrombus removal rate which is lower than contemporary studies with clearance rates greater than $90 \%$ with both CDT and PMT. Potential explanations for the lower success rate in the ATTRACT trial are operator unfamiliarity with PMT devices, variation in technique (50 hospitals within the trial), and lower doses of tissue plasminogen activator (tPA). Investigators were limited to 30 -hour infusions or $35 \mathrm{mg}$ total of tPA whereas those in the CaVenT trial used CDT only and capped at 96 hours. Furthermore, only 39\% of the intervention arm within the ATTRACT trial received stents, some of which were atypically small in diameter. Recent studies have reported poor thrombus clearance and unstented disease as the strongest predictors of lysis failure. Experts in venous intervention have since shifted to more aggressive early PMT with liberal use of large diameter stents as data from the national venous registry reported better patency in limbs treated with stents compared to those without-yet another way that the ATTRACT trial varies from contemporary venous practices (6-8).

Lastly, venous experts debate whether the Villalta score is an effective measure of PTS. Per some critics, the Villalta score is sensitive but not specific, therefore the binary use of this scale is not necessarily a meaningful tool. This is further supported by a difference in the development of PTS when using the Venous Clinical Severity Score (VCSS). When defining PTS as a VCSS score $\geq 4$, there is a significant difference between PMT/CDT and control groups $(30 \%$ vs. $40 \%, \mathrm{P}<0.05)$. As mentioned previously, when treating the Villalta score as a "continuous" measure as a way to quantify PTS severity, a significant difference in favor of intervention over anticoagulation was identified. And finally, the ATTRACT trial has also been criticized for evaluating patients too early as PTS is generally not evident until after 2 years (4). This may falsely decrease the proportion of patients diagnosed with PTS. The field of venous disease would benefit greatly if the ATTRACT investigators continued their work and published 5-year results.

Despite its limitation, the ATTRACT trial provides the venous community with the largest randomized sample of patients with iliofemoral DVT. Although its results may point towards fewer than anticipated benefits for PMT/ CDT, its effect on immediate symptom relief, the reduction in severity of PTS, and its impact on the QOL for patients with iliofemoral DVT cannot be underestimated. This powerful and high quality study will likely be the basis of many clinicians' and interventionalists' practices for several years to come.

\section{Acknowledgments}

Funding: None.

\section{Footnote}

Conflicts of Interest: Both authors have completed the ICMJE uniform disclosure form (available at http://dx.doi. org/10.21037/cdt.2019.08.08). EDA has received speaking honoraria from Boston Scientific Corporation. CG has no conflicts of interest to declare.

Ethical Statement: The authors are accountable for all aspects of the work in ensuring that questions related to the accuracy or integrity of any part of the work are appropriately investigated and resolved.

Open Access Statement: This is an Open Access article distributed in accordance with the Creative Commons Attribution-NonCommercial-NoDerivs 4.0 International License (CC BY-NC-ND 4.0), which permits the noncommercial replication and distribution of the article with the strict proviso that no changes or edits are made and the original work is properly cited (including links to both the formal publication through the relevant DOI and the license). See: https://creativecommons.org/licenses/by-nc-nd/4.0/.

\section{References}

1. Vedantham S, Goldhaber SZ, Julian JA, et al. Pharmacomechanical catheter-directed thrombolysis for deep-vein thrombosis. N Engl J Med 2017;377:2240-52.

2. Comerota AJ, Kearon C, Gu CS, et al. Endovascular thrombus removal for acute iliofemoral deep vein thrombosis. Circulation 2019;139:1162-73.

3. Enden T, Haig Y, Kløw NE, et al. Long-term outcome after additional catheter-directed thrombolysis versus standard treatment for acute iliofemoral deep vein 
thrombosis (the CaVen T study): a randomised controlled trial. Lancet 2012;379:31-8.

4. O'Sullivan GJ, de Graaf R, Black SA. Just how attractive is the ATTRACT trial? Cardiovasc Intervent Radiol 2018;41:1313-7.

5. Avgerinos ED, Chaer RA. The ATTRACTiveness of catheter-directed thrombolysis. J Vasc Surg Venous Lymphat Disord 2018;6:303.

6. Avgerinos ED, Saadeddin Z, Abou Ali AN, et al. Outcomes and predictors of failure of iliac vein stenting after catheter-directed thrombolysis for acute iliofemoral

Cite this article as: Go C, Avgerinos ED. Iliofemoral lysis for DVT remains ATTRACTive. Cardiovasc Diagn Ther 2020;10(2):104-106. doi: 10.21037/cdt.2019.08.08 thrombosis. J Vasc Surg Venous Lymphat Disord 2019;7:153-61.

7. Mewissen MW, Seabrook GR, Meissner MH, et al. Catheter-directed thrombolysis for lower extremity deep venous thrombosis: report of a national multicenter registry. Radiology 1999;211:39-49.

8. Go C, Saadeddin Z, Pandya Y, et al. Single versus multiple-stage catheter-directed thrombolysis for acute iliofemoral DVT does not impact iliac vein stent length or patency rates. J Vasc Surg Venous Lymphat Disord 2019;7:abstr 301. 